\title{
Preface to the special issue on Mesoscopic Solar Cells
}

\author{
Michael Grätzel (凶) \\ Laboratoire de Photoniques et Interfaces, Institut des Sciences et Ingénierie Chimiques, \\ École Polytechnique Fédérale de Lausanne, Lausanne, Switzerland
}

(C) Higher Education Press and Springer-Verlag Berlin Heidelberg 2016

Energy crisis and environment pollution are the main challenges of human beings in the 21th century. Developing solar cells with high efficiency and stability at low production cost is an effective way to achieve "price parity" with fossil-fuel-based grid power and large-scale use of solar energy. Since the breakthrough of dye-sensitized solar cells (DSSCs) in 1991, mesoscopic solar cells have been developing fast. Especially in the past three years, the power conversion efficiency (PCE) of perovskite solar cells based on mesoporous structure increased at an amazing rate. Recently, a certificated PCE of $21.0 \%$ was reached, which is higher than the certificated record PCE of polycrystalline silicon solar cells, indicating a competitive advantage. However, as new type of photovoltaics, solar cells based on dyes, quantum dots or perovskite pigments as light harvesters, still have many unknowns to be explored. I am delighted to be invited by Frontiers of Optoelectronics, as the guest editor of the first issue in 2016, to arrange the special edition on Mesoscopic Solar Cells.

This issue contains 7 review and research papers, all invited written by international well known scientists who are active in the field of mesoscopic solar cells. It covers research frontiers from DSSCs, quantum-dot-sensitized solar cells to mesoscopic perovskite solar cells, from fabrication, characterization to optimization of different components of mesoscopic solar cells. This special issue offers a general view of the development of mesoscopic solar cells. One of the most important components in DSSCs is the sensitizing dye. The donor- $\pi$ bridge-acceptor (D- $\pi-A)$ structure is the mainstream in the design of organic sensitizers. Hua et al. discussed the donor design and modification strategies of metal-free sensitizers for highly-efficient DSSCs, however, some sensitizers that do not follow D- $\pi$-A structure also present good efficiency when applied in DSSC devices. Robertson and Hu summarized the atypical dyes in order to inspire more diverse designs towards highly efficient DSSCs. The effect of the perovskite morphology on the photovoltaic performance is a critical factor. Etgar and Cohen reviewed various parameters influence and control the organo-metal halide perovskite crystallization and morphology, such as the annealing process, the precursor solvent, anti-solvent treatment and additives to the perovskite solution. Except the comprehensive reviews of organic sensitizers in DSSC, some research results of mesoscopic solar cells with novel photoelectrode, sensitizers, and cathode layers were presented as well. Zhao et al. proposed a new architecture design, micro-scale hierarchical $\mathrm{TiO}_{2}$ nanowires, for nanowire-based quantum-dot-sensitized solar cells to improve the photovoltaic performance. Li et al. designed and synthesized a series of metal-free organic sensitizers with D-A'$\pi$-A configurations, in which quninoxaline or benzoxadiazole moiety was incorporated into the conjugated bridge as the auxiliary electron acceptor $\left(A^{\prime}\right)$ to extend the absorption spectra and broaden the light-harvesting region. Yang et al. explored the use of P3HT modified carbon nanotubes (CNTs@P3HT) (P3HT = poly(3-hexylthiophene)) for the cathodes of hole transporter free, mesoscopic perovskite solar cells, simultaneously achieving high-performance, high stability and low-cost perovskite solar cells. Despite of the increasing importance of $\mathrm{CH}_{3} \mathrm{NH}_{3} \mathrm{Pbl}_{3}$ based organicinorganic hybrid perovskites, studies relating to basic structural characterization of these powerful light-harvesting materials remains sparse. Padture et al. conducted a systematic ambient Raman spectroscopy characterization study of $\mathrm{MAPbl}_{3}$ thin films to elucidate the possible sources of artifacts in the Raman spectra, and raised the awareness of the challenges in the ambient Raman spectroscopy of $\mathrm{MAPbl}_{3}$ perovskites.

I would like to thank the authors for their excellent works, which set a new landmark in this rapidly evolving and

Received January 19, 2016

E-mail: michael.graetzel@epfl.ch 
fascinating field. I am particularly grateful to the strong support from Prof. Dexiu Huang and the assistance from Prof. Hongwei Han for this special issue.

Prof. Michael Grätzel

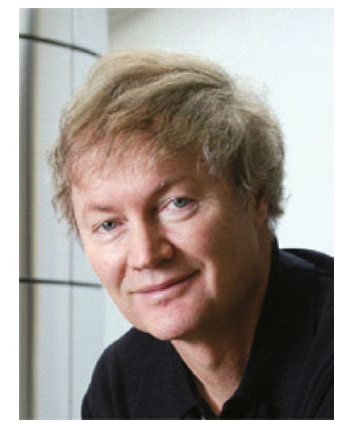

Michael Grätzel, Professor at the École Polytechnique Fédérale de Lausanne, he directs there the Laboratory of Photonics and Interfaces. He pioneered the use of mesoscopic materials in energy conversion systems, in particular photovoltaic cells, lithium ion batteries and photo-electrochemical devices for the splitting of water into hydrogen and oxygen by sunlight. He discovered a new type of solar cell based on dye sensitized nanocrystalline oxide films. Mass production has started in October 2009. Author of over 1000 publications, two books and inventor of more than 50 patents, his work has been cited over 148000 times (H-index 176) making him one of the 10 most highly cited chemists in the world.

He has received prestigious awards, including the 2014 Samson Prime Minister Prize, the Balzan Prize, the Galvani Medal, the Faraday Medal, the Harvey Prize, the Gerischer Award, the Dutch Havinga Award and Medal, the International Prize of the Japanese Society of Coordination Chemistry, the ENI-Italgas Energy-Prize and the year 2000 European Grand Prix of Innovation. His most recent awards include the 2012 Albert Einstein World Award of Science, 2011 Gutenberg Research Award, 2011 Paul Karrer Gold Medal and the 2010 Millenium Technology Grand Prize. He was selected by the Scientific American as one of the 50 top researchers in the world. He received a doctor's degree in Natural Science from the Technical University Berlin and honorary doctors degrees from 11 Universities (Univ. Liege, Belgium (2013); Rskilde Univ., Denmark (2012); Huazhong Univ of Sci. \& Tech., Wuhan, China (2011); Nangyang Tech. Univ (NTU), Singapore (2011); Univ. Lund, Sweden (2011); Univ. Nova Gorica, Slovenia (2010); Univ. Hasselt, Belgium (2009); Delft Univ. of Technology, Netherlands (2006); Univ. Turin (Torino), Italy (2004) and Univ. Uppsala, Sweden (1996)). He has been the Mary Upton Visiting Professor at Cornell University and a Distinguished Visiting Professor at the National University of Singapore. He was an Invited Professor at the University of Berkeley, the Ecole Nationale de Chachan (Paris) and Delft University of Technology. In 2009, he was named Distinguished Honorary Professor by the Chinese Academy of Sciences (Changchun) and the Huazhong University of Science and Technology. He is a member of the Swiss Chemical Society as well as of the European Academy of Science, a Fellow of the Royal Society of Chemistry and was elected honorary member of the Société Vaudoise des Sciences Naturelles and the Bulgarian Academy of Science. 